\title{
Fine Structure of the QCD String Spectrum
}

\author{
K. Jimmy Juge \\ Institute for Theoretical Physics, University of Bern, Sidlerstrasse 5, CH-3012 Bern, Switzerland \\ Julius Kuti \\ Department of Physics, University of California at San Diego, La Jolla, California 92093-0319 \\ Colin Morningstar \\ Department of Physics, Carnegie Mellon University, Pittsburgh, PA 15213, USA
}

(Dated: July 10, 2002)

\begin{abstract}
Using advanced lattice methods in Quantum Chromodynamics, three distinct scales are established in the excitation spectrum of the gluon field around a static quark-antiquark pair as the color source separation $R$ is varied. On the shortest length scale, the excitations are consistent with states created by local gluon field operators arising from a multipole operator product expansion. An intermediate crossover region below $2 \mathrm{fm}$ is identified with a dramatic rearrangement of the level orderings. On the largest length scale of 2-3 fm, the spectrum agrees with that expected for string-like excitations. The energies nearly reproduce asymptotic $\pi / R$ string gaps, but exhibit a fine structure, providing important clues for developing an effective bosonic string description.
\end{abstract}

PACS numbers: 11.15.Ha, 11.25.Pm, 12.38.Aw, 12.38.Gc

There exists great interest and considerable effort to explain quark confinement in Quantum Chromodynamics from the string theory viewpoint. The ideas of 't Hooft, Polyakov, Witten, and others, and recent glueball spectrum and string tension calculations in AdS theories are illustrative examples. In a somewhat complementary approach, the search for a microscopic mechanism to explain quark confinement in the QCD vacuum continues with vigorous effort. Our view is that the relevant properties of the underlying effective QCD string theory, whether it emerges from strictly string theoretic ideas or from the microscopic theory of the vacuum, are coded in the excitation spectrum of the confining flux. To establish the main features of this spectrum is the objective of this work.

We report here, for the first time, our comprehensive determination of the rich low-lying energy spectrum of the excited gluon field between a static quarkantiquark $(q \bar{q})$ pair in the fundamental color representation of $S U(3)_{c}$. Only a few of these states have been previously studied and only for $q \bar{q}$ separations $R$ up to 1 fm [1, 2, 3]. Due to our use of recent advances in lattice gauge theory technology, which include anisotropic lattices, an improved lattice gauge action, and large sets of creation operators, we have been able to determine the excitation spectrum for a large range of $R$ values from 0.1 to $3 \mathrm{fm}$, and for a greatly extended set of excited states never studied before. To investigate the onset of string-like behavior when the flux is much longer than its intrinsic width became a realistic goal [4]. The effects of light $q \bar{q}$ pairs in the vacuum are not included in this study, with focus on the confining properties of the gluon field. Earlier results from less extensive simulations were reported in Ref. [5].
Three exact quantum numbers which are based on the symmetries of the problem determine the classification scheme of the gluon excitation spectrum in the presence of a static $q \bar{q}$ pair. We adopt the standard notation from the physics of diatomic molecules and use $\Lambda$ to denote the magnitude of the eigenvalue of the projection $\mathbf{J}_{g} \cdot \hat{\mathbf{R}}$ of the total angular momentum $\mathbf{J}_{g}$ of the gluon field onto the molecular axis with unit vector $\hat{\mathbf{R}}$. The capital Greek letters $\Sigma, \Pi, \Delta, \Phi, \ldots$ are used to indicate states with $\Lambda=0,1,2,3, \ldots$, respectively. The combined operations of charge conjugation and spatial inversion about the midpoint between the quark and the antiquark is also a symmetry and its eigenvalue is denoted by $\eta_{C P}$. States with $\eta_{C P}=1(-1)$ are denoted by the subscripts $g(u)$. There is an additional label for the $\Sigma$ states; $\Sigma$ states which are even (odd) under a reflection in a plane containing the molecular axis are denoted by a superscript $+(-)$. Hence, the low-lying levels are labeled $\Sigma_{g}^{+}, \Sigma_{g}^{-}$, $\Sigma_{u}^{+}, \Sigma_{u}^{-}, \Pi_{g}, \Pi_{u}, \Delta_{g}, \Delta_{u}$, and so on. For convenience, we use $\Gamma$ to denote these labels in general.

The gluon excitation energies $E_{\Gamma}(R)$ were extracted from Monte Carlo estimates of generalized large Wilson loops using extended sets of 15 to 25 operators, projected onto the various symmetry sectors, on three-dimensional time slices of the lattice. Monte Carlo estimates of the $W_{\Gamma}^{i j}(R, t)$ Wilson correlation matrices were obtained in a series of simulations performed on our dedicated UP2000 Alpha cluster using an improved gauge-field action [6]. The couplings $\beta$, input aspect ratios $\xi$, and lattice sizes for each simulation will be detailed in forthcoming publications but are available now upon request. The use of anisotropic lattices, with the temporal lattice spacing $a_{t}$ much smaller than the spatial spacing $a_{s}$, was crucial for resolving the gluon excitation spectrum, particularly for 
large $R$. The renormalized anisotropy was determined using the torelon dispersion relation. To hasten the onset of asymptotic behavior in effective mass plots, iterativelysmeared spatial links were used in the generalized Wilson loops whose temporal segments were constructed from thermally-averaged links to reduce statistical noise.

Restricted to the $R=0.2-3 \mathrm{fm}$ range of a selected simulation, energy gaps $\Delta E$ above the ground state are compared to asymptotic string gaps for 18 excited states in Fig. 1] The quantity $\Delta E /(N \pi / R)-1$ is plotted to show percentage deviations from the asymptotic string levels for string quantum number $N=1,2,3,4$. The energy gaps, far below the null lines of the plots and strongly split at fixed $N$, differ from the simple string gaps for $R<2 \mathrm{fm}$ and a fine structure remains visible beyond $R=2 \mathrm{fm}$. The three relevant scales of the spectrum are discussed below.

The errors shown in Fig. 1 are statistical only and were determined using standard bootstrap techniques. Autocorrelations were explicitly calculated to ensure that the statistical errors were not underestimated. Dependence of the error estimates on the choice of temporal fit ranges was minimized by using fits to sums of two exponentials. The removal of contamination in our results from excited-states was checked using a variety of methods, including Bayesian constrained fits to large sums of exponentials, variational re-diagonalizations, and correlation matrix fits to sums of coupled exponentials. The insignificance of systematic effects from finite volume was verified, and simulations carried out for several other lattice spacings indicate that finite spacing errors in the results shown in Fig. 1 are expected to be small.

Short distance spectrum. For $R \ll 1 \mathrm{fm}$, the observed level ordering is consistent with the short-distance physics of gluon field excitations which are trapped around a dominantly color octet static $q \bar{q}$ pair. Since gluon field dynamics will only depend softly on $\mathbf{R}$, a new approximate symmetry is expected in the $R \sqrt{\sigma} \ll 1$ limit (the string tension $\sigma$ sets the scale of transverse dimension across the flux). This symmetry was first noted within the context of the static bag picture of gluon excitations [7] and used more extensively in Refs. [4, 8, 9, 10]. In particular, it was shown in Ref. [10] how the shortdistance operator product expansion of gluon excitations around static color sources, in lowest order of the multipole expansion of the gluon field, can be applied to the spectrum yielding similar results to the bag picture [8] without phenomenological model assumptions.

Gluon excitations will transform according to representations of $O(3)$ in the short-distance limit so that $\Lambda$ in the set $\Gamma$ will be replaced by the gluon field angular momentum $J$. A representation with fixed $J$ contains degenerate states with different $\Lambda$ values. For illustration, gluon field operators of $O(3)$ multiplets are listed in Table 【 for ten states with their short distance angular momentum quantum number $J$ for each multiplet. There
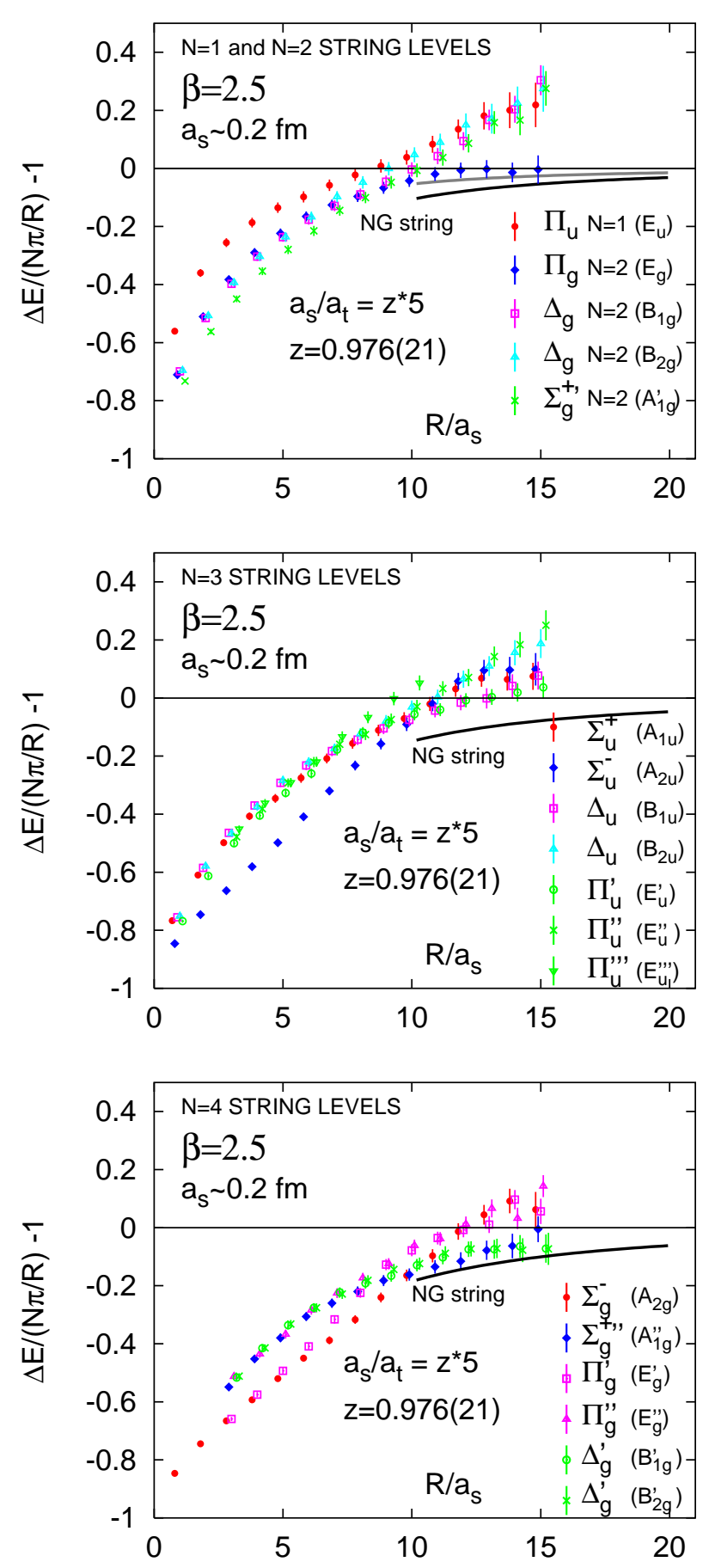

FIG. 1: Energy gaps $\Delta E$ above $\Sigma_{g}^{+}$are shown in string units for quantum numbers in continuum and lattice notation. The Nambu-Goto string is discussed in the text.

are two triplets $\left(\Sigma_{g}^{+^{\prime}}, \Pi_{g}\right),\left(\Sigma_{u}^{-}, \Pi_{u}\right)$, and two $J=2$ multiplets $\left(\Sigma_{g}^{-}, \Pi_{g}^{\prime}, \Delta_{g}\right),\left(\Sigma_{u}^{+}, \Pi_{u}^{\prime}, \Delta_{u}\right)$ in Table $\Pi$ and $\Delta$ states occur with two opposite sign chiralities $\Lambda$ within each $O(3)$ multiplet. Within the degenerate multiplets, one finds different string quantum numbers $N$ mixed together. Fig. 2 illustrates the remarkable working of the 
TABLE I: Operators to create excited gluon states for small $q \bar{q}$ separation $R$ are listed. $\mathbf{E}$ and $\mathbf{B}$ denote the electric and magnetic operators, respectively. The covariant derivative $\mathbf{D}$ is defined in the adjoint representation [10].

\begin{tabular}{lll}
\hline \hline gluon state & $J$ & operator \\
\hline$\Sigma_{g}^{+\prime}$ & 1 & $\mathbf{R} \cdot \mathbf{E}, \quad \mathbf{R} \cdot(\mathbf{D} \times \mathbf{B})$ \\
$\Pi_{g}$ & 1 & $\mathbf{R} \times \mathbf{E}, \quad \mathbf{R} \times(\mathbf{D} \times \mathbf{B})$ \\
\hline$\Sigma_{u}^{-}$ & 1 & $\mathbf{R} \cdot \mathbf{B}, \quad \mathbf{R} \cdot(\mathbf{D} \times \mathbf{E})$ \\
$\Pi_{u}$ & 1 & $\mathbf{R} \times \mathbf{B}, \quad \mathbf{R} \times(\mathbf{D} \times \mathbf{E})$ \\
\hline$\Sigma_{g}^{-}$ & 2 & $(\mathbf{R} \cdot \mathbf{D})(\mathbf{R} \cdot \mathbf{B})$ \\
$\Pi_{g}^{\prime}$ & 2 & $\mathbf{R} \times(\mathbf{R} \cdot \mathbf{D}) \mathbf{B}+\mathbf{D}(\mathbf{R} \cdot \mathbf{B}))$ \\
$\Delta_{g}$ & 2 & $(\mathbf{R} \times \mathbf{D})^{i}(\mathbf{R} \times \mathbf{B})^{j}+(\mathbf{R} \times \mathbf{D})^{j}(\mathbf{R} \times \mathbf{B})^{i}$ \\
\hline$\Sigma_{u}^{+}$ & 2 & $(\mathbf{R} \cdot \mathbf{D})(\mathbf{R} \cdot \mathbf{E})$ \\
$\Pi_{u}^{\prime}$ & 2 & $\mathbf{R} \times(\mathbf{R} \cdot \mathbf{D}) \mathbf{E}+\mathbf{D}(\mathbf{R} \cdot \mathbf{E}))$ \\
$\Delta_{u}$ & 2 & $(\mathbf{R} \times \mathbf{D})^{i}(\mathbf{R} \times \mathbf{E})^{j}+(\mathbf{R} \times \mathbf{D})^{j}(\mathbf{R} \times \mathbf{E})^{i}$ \\
\hline \hline
\end{tabular}

predicted short-distance degeneracies. Only the states $\Delta_{u}$ and $\Sigma_{g}^{+}$show considerable soft breaking of the approximate symmetry at the shortest $R$ values.

Crossover region. For $0.5 \mathrm{fm}<R<2 \mathrm{fm}$, a dramatic crossover of the energy levels toward a string-like spectrum as $R$ increases is observed. For example, the states $\Sigma_{u}^{-}$with $N=3$ and $\Sigma_{g}^{-}$with $N=4$ break violently away from their respective short-distance $O(3)$ degeneracies to approach the ordering expected from bosonic string theory near $R \sim 2 \mathrm{fm}$.

An interesting feature of the crossover region is the successful parametrization of the $\Sigma_{\mathrm{g}}^{+}$ground state energy by the empirical function $E_{0}(R)=a+\sigma R-c \frac{\pi}{12 R}$, with the fitted constant $c$ close to unity, once $R$ exceeds $0.5 \mathrm{fm}$. The Casimir energy of a thin flux line was calculated in Refs. 11, 12], yielding $c=1$, and this approximate agreement is often interpreted as evidence for string formation. While the spectrum, including the qualitative ordering of the energy levels, differs from the naive bosonic string gaps for $R<1 \mathrm{fm}$, a high precision calculation shows the rapid approach of $c_{\text {eff }}(R)$ to the asymptotic Casimir value in the same $R$ range [13. Although there is no inconsistency between the two different findings, a deeper understanding of this puzzling situation is warranted.

We will return to this issue in a high precision study of the 3-dimensional $Z(2)$ gauge model in a future publication [14]. This accurate study of $c_{\text {eff }}(R)$ and the excitation spectrum of the $Z(2)$ flux line for a wide range of $R$ values between $0.3 \mathrm{fm}$ and $10 \mathrm{fm}$ will clearly demonstrate the early onset of $c \approx 1$ without a well-developed string spectrum. For now, Fig. [3]shows the lowest excitations in $Z(2)$ for $R=0.7 \mathrm{fm}$, revealing a bag-like disorder profile surrounding the static $q \bar{q}$ pair in the vacuum [14]. The two lowest energy levels are substantially dislocated from exact $\pi / R$ string gaps and all other excitations form a continuous spectrum above the glueball threshold. Since the submission of this work, a new study of $Z(2)$ at finite temperature has appeared [15], reporting very early onset of string behavior in support of Ref. 13.

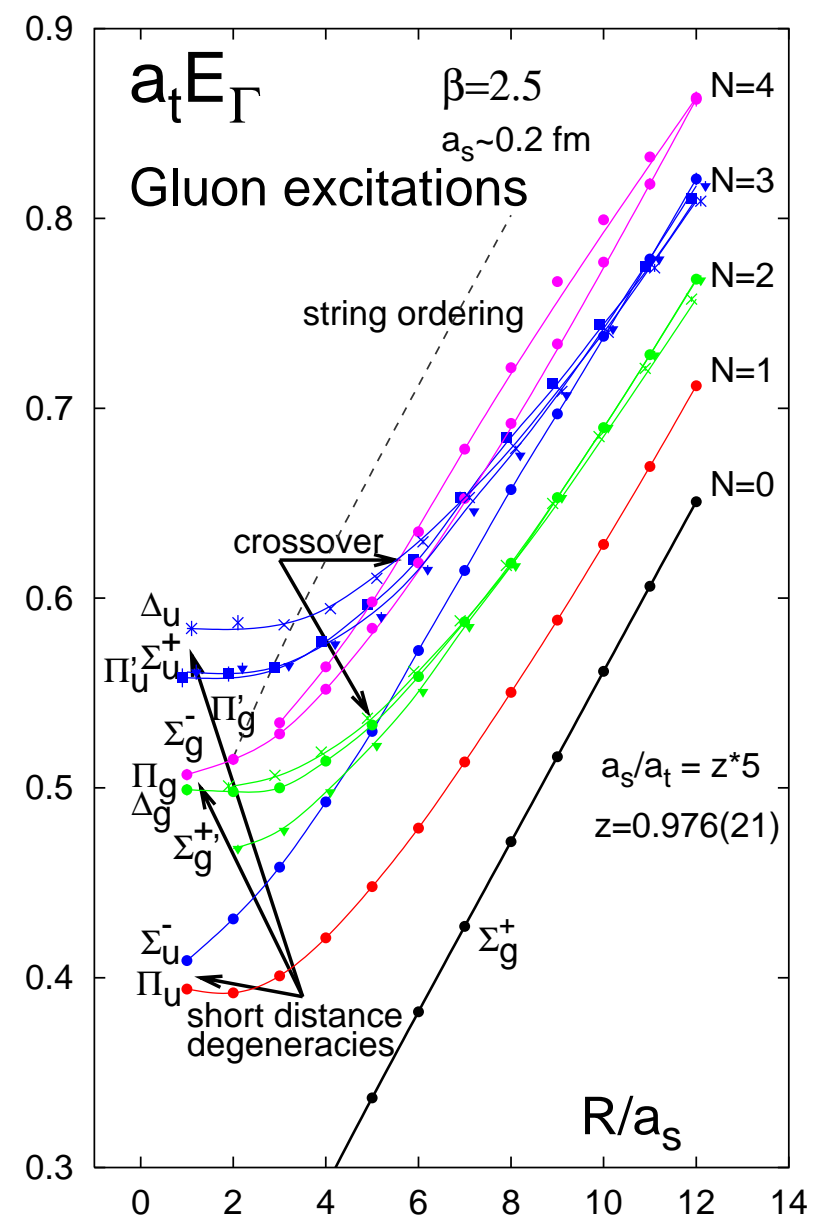

FIG. 2: Short-distance degeneracies and crossover in the spectrum. The solid curves are only shown for visualization. The dashed line marks a lower bound for the onset of mixing effects with glueball states which requires careful interpretation.

String limit. For $R>2 \mathrm{fm}$, the energy levels exhibit, without exception, the ordering and approximate degeneracies of string-like excitations. The levels nearly reproduce the asymptotic $\pi / R$ gaps, but an intriguing fine structure remains.

It has been anticipated that the interactions of massless excitations on long flux lines are described by a local derivative expansion of a massless vector field $\boldsymbol{\xi}$ with two transverse components in four-dimensional spacetime 11, 12]. Symmetries of the effective QCD string Lagrangian require a derivative expansion of the form

$$
\mathcal{L}_{\text {eff }}=a \partial_{\mu} \boldsymbol{\xi} \cdot \partial_{\mu} \boldsymbol{\xi}+b\left(\partial_{\mu} \boldsymbol{\xi} \cdot \partial_{\mu} \boldsymbol{\xi}\right)^{2}+c\left(\partial_{\mu} \boldsymbol{\xi} \cdot \partial_{\nu} \boldsymbol{\xi}\right)\left(\partial_{\mu} \boldsymbol{\xi} \cdot \partial_{\nu} \boldsymbol{\xi}\right)+\ldots,
$$

where the dots represent further terms with four or more derivatives in world sheet coordinates. The coefficient $a$ has the dimension of a mass squared and can be identified with the string tension $\sigma$. The other coefficients must be determined from the underlying microscopic theory. Examples with calculable coefficients include the $D=3 Z(2)$ 


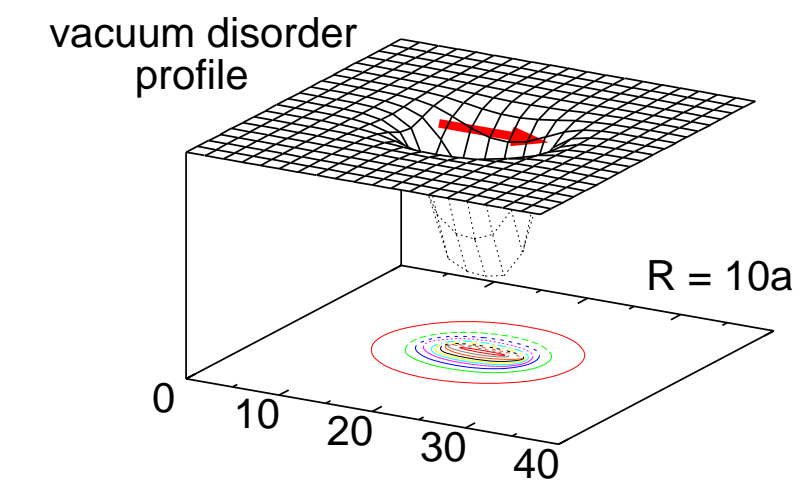

1 st excitation

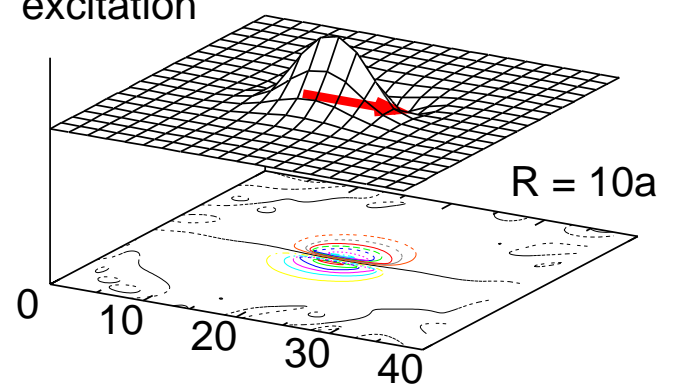

2
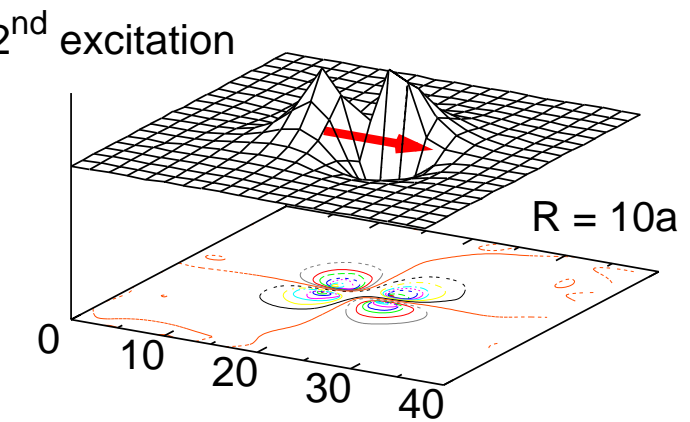

FIG. 3: The disorder profile of the vacuum and two excitations are shown with arrows indicating the $q \bar{q}$ axis.

defect line and the $D=4$ Nielsen-Olesen vortex in a semiclassical expansion of field theory. Similar calculations in QCD would require a quantitative understanding of quark confinement.

Asymptotic string gaps and the observed fine structure of the spectrum provide the most direct tests of effective QCD string formation for large $R$. Low frequency excitations are dominated by the first term which describes the asymptotic spectrum of massless modes with exact $\pi / R$ string gaps. Higher dimensional operators are expected to generate perturbative fine structure corrections in higher powers of $R^{-1}$. Matching the coefficients of Eq. (1) to the spectrum is a realistic goal for future high precision simulations. Since it is reasonable to expect that the first few coefficients arise from the geometric properties of the effective string, it is useful to introduce the Nambu-Goto (NG) action $\sigma \sqrt{1+\partial_{\mu} \boldsymbol{\xi} \cdot \partial_{\mu} \boldsymbol{\xi}}$ as the simplest geometric model. The NG spectrum with fixed end boundary conditions in $D$ dimensions was first calculated in Ref. [16] with the result $E_{N}=\sigma R\left(1-\frac{D-2}{12 \sigma R^{2}} \pi+\frac{2 \pi N}{\sigma R^{2}}\right)^{\frac{1}{2}}$. Although there exists a quantization problem in angular momentum rotations around the $q \bar{q}$ axis at finite $R$ values unless $D=26$ [16], the problem disappears in the large $R$ limit. It has been widely expected that predictions at large $R$ from the NG model will be similar to effective bosonic string theory with a good description of the observed spectrum. Deviations from the NG string at large $R$ in Fig. 1 may indicate rigidity and other missing physical properties of the flux line in the model.

In this work, striking confirmation of string-like flux formation of the gluon field for large $q \bar{q}$ separations was presented. Our simulations revealed a first glimpse of a fine structure in the large-distance spectrum, offering the tantalizing possibility of understanding the nature of the QCD string in future high precision simulations.

Throughout this extended project, we benefited from discussions with M. Peardon, G. Bali, R. Brower, K. Intriligator, P. Hasenfratz, M. Lüscher, C. Michael, S. Necco, F. Niedermayer, R. Sommer, J. Soto, P. Weisz, and many others. This work was supported by the DOE, Grant No. DE-FG03-97ER40546, the NSF under Award PHY-0099450 and the European Community's Human Potential Programme under contract HPRN-CT-200000145, Hadrons/Lattice QCD.

[1] I.J. Ford, R.H. Dalitz, and J. Hoek, Phys. Lett. B 208, 286 (1988).

[2] S. Perantonis and C. Michael, Nucl. Phys. B347, 854 (1990) .

[3] G. Bali et al., Phys. Rev. D 62, 054503 (2000); G. Bali, Phys. Rept. 343, 1 (2001).

[4] K.J. Juge, J. Kuti, and C. Morningstar, From surface roughening to $Q C D$ string theory, in Proceedings of the 24th Johns Hopkins Workshop, 143 (2000) and hep-lat/0103008 Nucl. Phys. (Proc. Suppl.) B83, 503 (2000); B106, 691 (2002).

[5] K.J. Juge, J. Kuti, and C. Morningstar, Nucl. Phys. (Proc. Suppl.), B63, 326 (1998); B73, 590 (1999).

[6] C. Morningstar and M. Peardon, Phys. Rev. D 56, 4043 (1997).

[7] P. Hasenfratz, R. Horgan, J. Kuti, and J. Richard, Phys. Lett. B95, 299 (1980).

[8] K.J. Juge, J. Kuti, and C. Morningstar, Nucl. Phys. (Proc. Suppl.) B63, 543 (1998).

[9] M. Foster and C. Michael, Phys. Rev. D 59094509 , (1999).

[10] N. Brambilla, A. Pineda, J. Soto, and A. Vairo, Nucl. Phys. B566, 275 (2000).

[11] M. Lüscher, K. Symanzik, P. Weisz, Nucl. Phys. B173, 365 (1980).

[12] M. Lüscher, Nucl. Phys. B180, 317 (1981).

[13] M. Lüscher and P. Weisz, hep-lat/0207003

[14] K.J. Juge, J. Kuti, and C. Morningstar, unpublished.

[15] M. Caselle, M. Hasenbusch, and M. Panero, hep-lat/0211012 
[16] J. F. Arvis, Phys. Lett. 127B, 106 (1983). 